\title{
Appendix: Finite prolongation in RGD-systems
}

\author{
Bernhard Mühlherr
}

\section{Introduction}

In this appendix we give the proof of Proposition 2.17 of [4]. It is a consequence of Theorem 4.5 below. The key step in our reasoning is based on a refinement of the arguments in the proof of Theorem 1.5 in [5]. The latter says that the set of chambers opposite a given chamber in a 2-spherical twin building is connected, if this condition holds in all rank 2 residues. Our refinement consists of giving a bound for the distance between two chambers in the set of opposite chambers depending on their distance in the building. In order to do this we have to strengthen the local condition on the rank two residues. This results in our somewhat technical Condition $(c o)_{k}$ below. It is almost always satisfied and more explanations are given in the final section of this note.

\section{Preliminaries}

Let $(W, S)$ be a Coxeter system and let $\ell: W \rightarrow \mathbf{N}$ be its associated length function. Let $\mathcal{B}=(\mathcal{C}, \delta)$ be a building of type $(W, S)$. For two chambers $c, d \in \mathcal{C}$ we put $\ell(c, d):=\ell(\delta(c, d))$.

Definition 2.1. A codistance on $\mathcal{B}$ is a mapping $\delta_{*}: \mathcal{C} \rightarrow W$ such that the following is satisfied for all $s \in S$ and $c \in \mathcal{C}$ where $w:=\delta_{*}(c)$.

(CD1) If $d \in \mathcal{C}$ is $s$-adjacent to $c$, then $\delta_{*}(d) \in\{w, w s\}$.

(CD2) If $\ell(w s)=\ell(w)+1$, then there exists a unique chamber $d$ which is $s$-adjacent to $c$ such that $\delta_{*}(d)=w s$.

Let $\delta_{*}$ be a codistance on $\mathcal{B}$. Then we put $\delta_{*}^{\text {op }}:=\left\{c \in \mathcal{C} \mid \delta_{*}(c)=1_{W}\right\}$. 




For $m>4$ there are free constructions by Abramenko ([1, Proposition 9 in Section II.2]) which show that one cannot expect similar results without further assumptions. His construction could probably even be modified to construct polygons satifying Condition (co) without satisfying ( $d c o$ ) for any $d \in \mathbf{N}$. Since we are only interested in twin buildings associated with $R G D$-systems, all rank 2 residues are Moufang. Thus, in view of the lemma above, we are left with the Moufang hexagons and Moufang octagons. In [2] it is shown that they satisfy Condition $(c o)$ in almost all cases. A natural strategy to establish (dco) for the Moufang hexagons and octagons is to analyze the proofs of Condition (co) for those polygons given in [2]. P. Abramenko and H. Van Maldeghem are convinced that this is indeed possible. They expect that Moufang hexagons with Condition (co) satisfy Condition (12co); apparently the octagons are a bit more complicated. The author thanks them for providing these informations about this question.

\section{References}

[1] P. Abramenko, Twin buildings and applications to S-arithmetic groups, Lecture Notes in Mathematics, vol. 1641, Springer, 1996.

[2] P. Abramenko and H. Van Maldeghem, Connectedness of opposite-flag geometries in Moufang polygons, European J. Combin. 20 (1999), 461468.

[3] A. Devillers, B. Mühlherr and H. Van Maldeghem, Codistances of 3-spherical buildings, Math. Ann. 354 (2012), 297-329.

[4] T. Hartnick, R. Köhl, A. Mars, On topological twin buildings and topological split Kac-Moody groups, Innov. Incidence Geom. 13 (2013), 1-71.

[5] B. Mühlherr and M. Ronan, Local to global structure in twin buildings, Invent. Math. 122 (1995), 71-81.

[6] H. Van Maldeghem, Generalized polygons, Monographs in Mathematics, vol. 93, Birkhäuser Verlag, Basel, 1998.

Bernhard Mühlherr

Universität Giessen, Mathematisches Institut, Arndtstrasse 2, 35392 Giessen, Germany

e-mail: bernhard.m.muehlherr@math.uni-giessen.de 